\title{
La construction de l'interpersonnalité chez Kant: une application juridique et éthique
}

\author{
Adelino Braz ${ }^{*}$ \\ Agregado para la cooperación educativa \\ Embajada de Francia en Colombia
}

Contre les accusations de formalisme et de monologisme qui frappent la philosophie kantienne, le concept d'interpersonnalité apporte une nouvelle lecture qui montre comment le projet kantien consiste à penser une coexistence des libertés. En démontrant que la substance se présente comme un être ouvert à l'existence et qu'elle n'acquiert ses déterminations qu'au sein d'une influence mutuelle entre les phénomènes, Kant confère à sa philosophie normative un horizon dans lequel, la personnalité s'inscrit dans le temps au sein d'une réciprocité de personnes. Il s'agit bien plutôt d'une interpersonnalité que d'une intersubjectivité, dans la mesure où ce sont des personnes exerçant leur liberté. Cette réciprocité, pensée par analogie avec l'égalité d'action et de réaction, trouve précisément son application dans le droit et dans l'éthique, de façon à constituer une coexistence des libertés extérieures et intérieures.

Mots clés : Substance, personne, communauté, droit, éthique.

\section{LA CONSTRUCCIÓN DE LA INTERPERSONALIDAD EN KANT: UNA APLICACIÓN JURÍDICA Y ÉTICA}

Contra las acusaciones de formalismo y de monologuismo que sacuden a la filosofía kantiana, el concepto de interpersonalidad aporta una nueva lectura que muestra cómo el proyecto kantiano consiste en pensar una coexistencia de las libertades. Demostrando que la substancia se presenta como un ser abierto a la existencia y que no adquiere sus determinaciones sino en el seno de una influencia mutua entre los fenómenos, Kant confiere a su filosofía normativa un horizonte en el cual la personalidad se inscribe en el tiempo, en el seno de una reciprocidad de personas. Se trata más bien de una interpersonalidad que de una intersubjetividad, en la medida en que son personas ejerciendo su libertad. Esta reciprocidad, pensada por analogía con la igualdad de acción y de reacción, encuentra precísamente su aplicación en el derecho y en la ética, con el objeto de constituir una coexistencia de libertades exteriores e interiores.

Palabras clave : Substancia, persona, comunidad, derecho, ética.

* Docteur en Philosophie de l’Université de Paris I, Panthéon-Sorbonne. Actualmente es Agregado para la cooperación educativa de la Embajada de Francia en Colombia. 
$\mathrm{D}$ eux reproches majeurs ayant une longue tradition, se dégagent dans les travaux portant sur la philosophie pratique kantienne. D'une part, cette philosophie normative est accusée de formalisme : la loi morale comme principe déterminant de l'action se présente à lêtre naturel raisonnable sous la forme d'un impératif catégorique, autrement dit comme une norme indépendante de tout contenu empirique qui, en s'imposant de façon inconditionnelle, permet à la volonté morale de se déterminer par pur respect pour le principe a priori de la loi morale, sans déterminer le contenu de l'action. D'autre part, cette législation universelle a priori de la raison est réduite dans la plupart des cas à un monologisme : la loi morale se présentant comme un fait de la raison (Faktum der vernunft), implique en premier lieu un rapport de soi à soi, et plus précisément une relation entre l'être sensible, phénoménal, et l'être intelligible, nouménal, excluant ainsi toute dimension communautaire. Or, cette interprétation semble contredire la véritable portée de cette exigence normative. En effet, comme nous indique l'auteur de la Métaphysique des mours, penser les lois du devoir revient en premier lieu à considérer un monde intelligible, au sein duquel, selon l'analogie avec l'univers physique, la liaison des êtres raisonnables, dans leurs rapports extérieurs s'opère par attraction (Anziehung) et répulsion $(A b s t o ß u n g){ }^{1}$. La difficulté qu'il s'agit de résoudre sur ce point est de savoir comment cette législation morale de sa propre raison peut être au fondement d'une relation entre les personnes, considérées comme êtres libres. Ce qui implique que non seulement il est nécessaire de légitimer un concept tel que celui d'interpersonnalité et de le distinguer d'une simple intersubjectivité, mais également de comprendre comment ce concept normatif est mis en œuvre dans les champs juridique et éthique à partir de la législation morale. Cette nouvelle lecture, pour être rigoureuse, doit d'abord envisager comment Kant envisage la substance, afin de cerner la nature de la personnalité et de la réciprocité des personnes, et de l'appliquer ensuite à la communauté juridique et à la communauté éthique. Ce qui est en jeu ici, c'est une redéfinition exacte du formalisme kantien et de sa dimension normative communautaire.

\section{Substance et communauté}

Le projet kantien consiste à poser dès l' « Amphibologie des concepts de la réflexion »² une question d'origine en discernant une différence entre la sensibilité et de l'entendement, ce qui entraîne une distinction dans le type de rapport au réel. Cette démarche transcendantale aboutit à un double résultat: d'une part, en montrant que notre nature est telle que les objets ne peuvent nous être donnés que sous le mode de l'intuition sensible, à savoir dans la sensibilité comprise comme réceptivité, le concept d'un noumène se révèle problématique car il explique une possibilité seulement logique dans lequel l'objet intelligible est seulement pensé par des catégories pures sans aucun schème de la sensibilité ${ }^{3}$. Ceci implique que la substance, dont les déterminations ne se rapportent qu'à sa réalité intérieure,

1 Métaphysique des mœurs, Ak. VI 449, p. 741. Oeuvres philosophiques d' Emmanuel Kant, sous la direction de Ferdinand Alquié, Bibliothèque de la Pléiade, Gallimard, Volumes I, II, III. Toutes les citations concernant cette édition indiquent la référence à l'édition de l'Académie de Berlin (Ak.), suivie de la page de l'édition française. Nous indiquons ici entre parenthèses le volume de l'édition Gallimard, pour chaque oeuvre, et les éditions pour les oeuvres qui ne sont pas publiées dans l'édition Gallimard.

2 Critique de la raison pure, Ak. III 215, A 260 / B 316, p. 998, (vol. I).

3 Ibid., Ak. III 229, A 286 / B 342, p. 1007.

La construcción de l'interpersonnalité chez Kant P. 60 - 68 
est un objet inconnaissable commenoumène. D'autre part, en tant que substantia phaenomenon, la substance ne peut être appréhendée que dans l'espace par les forces qui sont actives en lui, et plus rigoureusement sous la forme d'un phénomène inscrit dans des rapports: les déterminations intérieures ne se définissent plus indépendamment de toute condition extérieure, mais elles ne trouvent leur unité relative qu'au sein d'une interaction avec l'ensemble des réalités extérieures ${ }^{4}$. Il s'ensuit que la substance non seulement est indéterminée originairement, se révélant par là comme un être ouvert à l'existence, mais également qu'elle n'acquiert ses déterminations qu'au sein d'un dommage réciproque entre les phénomènes.

Or, l'enjeu d'une telle analyse est de fonder un concept de communauté à partir duquel s'exerce une influence réciproque des phénomènes les uns sur les autres. En effet, afin de ne pas instaurer une relation hétéronyme où la substance établirait à la fois une dépendance et une causalité, il est nécessaire de démontrer que l'unité du tout ne peut se fonder que sur une liaison réciproque dans laquelle chacun des termes corrélatifs est à l'égard de l'autre, à la fois déterminant et déterminé . Chaque substance contient la causalité de certaines déterminations dans une autre substance, et en même temps les effets de la causalité de l'autre, établissant ainsi un commerce réel des substances ${ }^{6}$. Ceci explique qu'un véritable commerce entre les substances est donc une relation homonyme où les substances sont liées par coordination. Précisons sur ce point, que ce commercium n'est pas admis sur le mode d'un commerce originaire qui s'expliquerait par la simple existence des substances, mais sur le mode d'un commerce dérivé et dynamique, leur principe de liaison est celui d'une influence physique réciproque. En ce sens, selon l'analyse de la troisième analogie ${ }^{7}$ la communauté (Gemeinschaft) est à la fois un commercium, une influence réciproque entre les phénomènes et une communio spatii, une communauté locale : l'unité du divers dans lequel les phénomènes forment un tout lié, est certes un effet du principe admis de communauté, mais c'est le commerce réel des substances qui est proprement le principe de la possibilité d'une connaissance empirique de la coexistence. Cela nous conduit à reconnaître que d'après cette première analyse, la substance, indéterminée originairement, se présente comme un être ouvert à l'existence qui n'acquiert ses déterminations que dans un influx physique, liant ainsi sa réalité à celle de la communauté dynamique. C'est précisément à partir de ce point que Kant fonde l'interpersonnalité, par la portée analogique qu'il confère au principe de communauté dans le domaine de la liberté.

4 «Les déterminations intérieures d'une substantia phaenomenon dans l'espace, reconnaît Kant ne sont que des rapports ; et elle-même n'est, en tout et pour tout, qu'un ensemble de pures relations ", Ibid., Ak. III 217, A 265 / B 321, p. 992.

5 «Le rapport des substances, dans lequel l'une contient des déterminations, dont le fondement est contenu dans l'autre, est le rapport d'influence, et quand réciproquement ce rapport contient le fondement des déterminations dans l'autre, il est rapport de communauté ou d'action réciproque ", ibid., Ak. III 181, A 211 / B 258, p. 943.

6 Ibid., Ak. III 183, A 212 / B 259, pp. 944-945.

7 «Les choses sont simultanées, lorsque dans l'intuition empirique la perception de l'une et celle de l'autre peuvent se suivre réciproquement ", ibid., Ak. III 180-181, A 211 / B 256-257, p. 942. Afin que cette simultanéité ne soit pas soumise à une simple contingence, il faut faire intervenir un concept de l'entendement capable d'indiquer que les objets sont réellement simultanés. Ce concept est celui de succession réciproque dans lequel chaque objet établit une influence réciproque avec un autre, inscrivant ainsi toutes les substances en une communauté universelle. 


\section{La réciprocité entre les personnes}

Cerner les composantes de cette construction revient d'abord à comprendre le passage du sujet à la personne, autrement dit du sujet ontologique au sujet pratique. L'indétermination originaire de la substance a pour conséquence de rendre le sujet, comme réalité ontologique, inconnaissable. Comme le souligne Kant dans la seconde édition des «Paralogismes de la raison pure », le sujet, dans sa propre représentation, ne prend pas conscience de lui-même comme déterminant, car tous les modes de conscience de soi ne sont que des fonctions logiques qui ne font connaître à la pensée aucun objet $t^{8}$ : la conscience de soi est seulement celle d'un moi déterminable en tant qu'elle ne peut être appréhendée que par le sens interne. Cette intuition intérieure, qui lie le divers sensible à l'unité de l'aperception, ne peut donc signifier l'identité de la personne comme substance. Cependant, si dans le domaine de la spéculation la personnalité se révèle stérile, elle rend intelligible l'idée d'un usage pratique de la raison en ce qu'elle permet de déterminer notre conduite « comme si notre destinée s'étendait infiniment au-delà de l'expérience et par conséquent de cette vie $»$. Cette remarque est d'importance, car en opérant un dépassement du temps historique, celui des séries causales empiriquement déterminées par une durée atemporelle, elle se situe à la racine même du passage à l'être humain comme être intelligible en se représentant une durée infinie (duratio noumenon) qui ne répond à aucun concept déterminable ${ }^{10}$ : ainsi, le sujet n'avance guère dans la connaissance de lui-même, mais pose une intention morale se poursuivant à l'infini, qui se maintient et demeure constamment la même. Il se conçoit comme principe de détermination qui choisit sa maxime comme si, à travers tous les changements, son état moral n'était soumis à aucune vicissitude temporelle. Par là, il devient possible pour le sujet de découvrir une spontanéité par laquelle sa réalité effective (unsere Wirklichkeit) devient déterminable indépendamment des conditions de l'intuition empirique, s'apercevant ainsi que « dans la conscience de notre existence, quelque chose $\mathrm{d}^{\prime}$ a priori est contenu » eu égard à un certain pouvoir interne, en relation à un monde intelligible ${ }^{11}$. C'est à partir de cette articulation que Kant parvient à concilier dans la seconde édition des paralogismes, la limitation du concept de personnalité comme objet de connaissance théorique et la possibilité d'une connaissance morale, ce qui permet de passer du sujet ontologique, inconnaissable, à la personne, sujet normatif. Il s'ensuit que la catégorie de la personnalité rend compte à la fois d'une cause intelligible imputable des actions appartenant au monde sensible, et d'une instance morale qui se donne à elle-même sa propre nature.

D'après le concept normatif de personnalité, Kant, dans la table des catégories de la liberté, et plus précisément dans la catégorie de la relation, reconstruit l'interpersonnalité ou la réciprocité entre personnes ${ }^{12}$. A ce titre, la catégorie de la relation pose une articulation

8 Critique de la raison pure, Ak. III 267, B 407, p. 1052. Dans la première édition des «Paralogismes de la raison pure », Kant mentionne un passage vers l'idée d'un usage pratique de la personnalité en précisant que ce concept ne procède à aucune extension de la connaissance de soi, mais s'avère nécessaire à l'usage pratique et seulement à celui-ci, ibid., Ak. IV 230, A 365-366, p. 1441.

9 Critique de la raison pure, Ak. III 275, B 421, p. 1062.

${ }^{10}$ La fin de toutes choses., Ak. VIII, p. 309 et Ak. VIII, p. 318, (vol. III).

11 Ibid., p. 1068.

12 Critique de la raison pratique, Ak. V 65, p. 687 (vol. II). 
entre la causalité nouménale, dont le principe se situe en dehors du monde sensible, et la réciprocité des personnes, selon une relation à la personnalité, à l'état de la personne et à la réciprocité d'une personne à l'état des autres. Dans ce passage, où les concepts de personne et de personnalité apparaissent pour la première fois ${ }^{13}$, la relation entre la personnalité et l'état de la personne, doit être comprise selon la loi de dépendance de l'effet par rapport à sa cause, ce qui correspond à la catégorie de la causalité ${ }^{14}$. Chaque action, comme phénomène, en tant qu'elle produit un événement, est elle-même quelque chose qui advient et qui présuppose un autre état où se rencontre sa cause. Cette dernière se présente soit comme sensible, ce qui renvoie à l'état de la personne empirique, physique ou psychique, relevant ainsi de conditions empiriques ; soit comme intelligible, auquel cas elle doit être rapportée à la nature morale que la personne se donne à elle-même, indépendamment des déterminations sensibles. Ainsi, l'état de la personne rend raison de tout changement d'action et permet par la-même de qualifier une action de bonne ou mauvaise en ce qu'il est nécessaire de recourir, au caractère de la maxime, compris comme effet de la condition morale de la personne ${ }^{15}$. Ce qui est pertinent dans ce passage, c'est l'inscription de l'action humaine dans le sensible, à partir d'une causalité intelligible, et l'horizon qu'elle ouvre sur la communauté humaine. Or, le dessein implicite de Kant dans les catégories de la liberté, est de montrer que la causalité entre la personnalité et l'état de la personne ne peut faire abstraction de l'interpersonnalité : autrement dit, l'action qui se manifeste comme événement établit une relation de l'individu à la communauté. En effet, le sujet historique agit dans le temps et rend raison de ses actions par sa dimension nouménale. Toutefois, ce sujet ne se réduit pas à une structure monologique qui ne renvoie qu' à une sphère privée de l'individu, sans aucun échange avec l'autre. Au contraire, parce que chaque action s'inscrit dans une relation réciproque d'une personne à l'état des autres, il est inscrit dans une dynamique dialogique ${ }^{16}$. La troisième catégorie selon laquelle toute action s'engage dans « une relation réciproque d'une personne à l'état des autres ${ }^{17}$ prend ainsi tout son sens : en appliquant la catégorie de la communauté d'action réciproque entre un agent et un patient ${ }^{18}$ à la sphère des personnes agissantes, tout être raisonnable conçoit son état ou son action dans un horizon d'interpersonnalité vivant en même temps ${ }^{19}$. Prise comme causalité intelligible qui se manifeste dans le temps, la personne influence l'état des autres personnes par une égalité d'action et réaction, selon le modèle mécanique de Newton, bien que cette interaction ne puisse être représentée concrètement dans aucune expérience.

L'analogie avec le modèle mécanique est décisive sur ce point pour comprendre le

${ }^{13}$ Le terme personne apparaît bien à la fin du deuxième scolie du $\S 8$, Ak. V 38, p. 655, mais cette occurrence n'est pas systématisée ni prise réellement en compte dans l'argumentation du passage. Notre remarque suit ici la note de G. Mohr «Personne, personnalité et liberté dans la Critique de la raison pratique ", Revue internationale de philosophie, mars 1988, vol. 42, n. 166, p. 308, note 33.

${ }^{14}$ Ibid., Ak. III 93, A 80 / B 105, p. 834.

15 Notons que l'expression « état de la personne » peut signifier soit l' «état (Zustand) moral », soit la maxime d'une personne d'après laquelle elle agit.

${ }^{16}$ Le terme dialogique est emprunté à la terminologie kantienne dans Métaphysique des mours, Ak. VI 478, et désigne un échange réciproque de questions et réponses entre le maître et l'élève, p. 775.

${ }_{17}$ Critique de la raison pratique, Ak. V 66, p. 689.

18 Critique de la raison pure, Ak. III 93, A 80 / B 105, p. 834.

${ }^{19}$ En effet, dès l'opuscule de 1766 à l'encontre de Swedenborg ${ }^{19}$, l'auteur réfute toute possibilité d'une communication entre des esprits immatériels, Rêves d'un visionnaire, Ak. II 322, pp. 533-535 (vol. I). 
fondement de l'interpersonnalité, dans la mesure où elle répond à une double fonction : montrer la nécessité d'une action réciproque dans la constitution de toute communauté, et instituer la limitation comme condition de toute coexistence des libertés. Le concept de communauté (Gemeinschaft) rend compte de cette réciprocité ou égalité d'action entre deux forces : il est possible de conclure à un effet $\mathrm{B}$ à partir d'une propriété de $\mathrm{A}$, et réciproquement, sans que cet effet, soit une propriété intrinsèque à celui qui en subit l'effet. Cette action fixe du coup la position de $B$ en l'excluant d'une zone déterminée de l'espace ${ }^{20}$. A la différence de la borne (Schranke) qui est une simple négation qui affecte une quantité dans la mesure où elle n'a pas de complétude absolue, la limite (Grenze) suppose toujours une frontière entre deux espaces. Afin de rendre compte de la réciprocité, la limitation doit être comprise à partir de la possibilité de la matière dans les Premiers principes métaphysiques de la science de la nature. La position de Kant est d'affirmer que la matière remplit un espace non par sa simple existence, mais par une force motrice particulière ${ }^{21}$. En effet, le concept même de matière est constitué par la force expansive, s'exerçant à partir de tous les points dans toutes les directions. L'élasticité est donc le fondement sur lequel repose le remplissement de l'espace, et qui qualifie originairement la matière. Or, pour une force d'extension donnée de la matière, il est possible de trouver une force de compression plus grande, obligeant ainsi la première à occuper un espace plus étroit. Toutefois, s'il est vrai que la matière peut être comprimée à l'infini, en aucun cas elle n'est entièrement pénétrée, si grande que soit la force de cette matière ${ }^{22}$. La limite réside donc dans cette impénétrabilité relative qui permet par l'action et la réaction de poser une unité dans l'espace. En supprimant cette limite, non seulement la réciprocité disparaît, mais l'espace lui-même devient impensable. A partir de cette analogie, il est possible de cerner la nature de la relation qui se joue dans l'interpersonnalité. D'une part, il s'agit d'une relation entre sujets certes, mais qui ne sont pas considérés du point de vue spéculatif mais du point de vue normatif. En effet, dans la mesure où cette relation concerne des êtres doués de liberté, des sujets pratiques dont les actions ne sont que l'effet d'une cause spontanée intelligible, cette réciprocité ne doit pas être comprise comme intersubjectivité, sous peine de ne pas considérer le domaine dans lequel elle s'inscrit, à savoir celui de la liberté. D'autre part, cette réciprocité se construit à partir de l'analogie avec le modèle de l'égalité d'action et de réaction, car la visée de Kant est de constituer une coexistence des libertés, une égalité d'actions libres entre personnes agissant dans le temps. Or, l'intérêt de cette construction est de trouver son application dans le droit et dans l'éthique, de façon à montrer que la morale ne se réduit pas à un monologisme mais bien à une communauté de libertés, ce qui du coup confère au formalisme kantien un nouveau sens.

\section{Application au droit et à l'éthique}

Cette analogie au fondement de l'interpersonnalité est établie tout d'abord avec le droit. Dans une note du §58 des Prolégomènes à toute métaphysique future qui pourra se présenter comme science, Kant montre la complète ressemblance qui existe entre le rapport juridique des actions

\footnotetext{
${ }^{20}$ Kant et la philosophie grecque et moderne, Actes du IIIème congrès de la société internationale d'études kantiennes de langue française, éd. par M. Moutsopoulos, Athènes, 1997.

${ }_{21}$ Premiers principes métaphysiques de la science de la nature, Ak. IV 497, p. 40, (vol. II).

22 Ibid., Ak. IV 501, p. 406.
} 
humaines et le rapport mécanique des forces motrices. De même que l'action est égale à la réaction en mécanique, dans le droit, «je ne puis jamais rien faire contre autrui sans lui donner le droit de faire la même chose contre moi dans les mêmes conditions ${ }^{23}$. Cependant, il convient de nuancer cette proposition afin d'en cerner la signification: si un homme a trompé ou menti, celui qui en est la victime ne commet pas d'injustice à son endroit en le trompant à son tour ; le premier ne peut sans doute pas se plaindre de l'action du second, mais il n'en demeure pas moins que ce dernier a agi injustement car son geste peut malgré tout causer une injustice générale. Il ne suffit donc pas de prétendre que l'on n'a causé aucun tort à autrui, pour légitimer son action, il faut de plus qu'elle soit compatible avec la liberté externe de chacun. En ce sens, pour saisir la portée de l'analogie, il se révèle indispensable de se reporter au $\S \mathrm{E}$ del" «Introduction à la Doctrine du droit » dans la Métaphysique des mours, dans lequel l'auteur explique que la loi d'une contrainte réciproque inhérente au droit s'accorde nécessairement avec la coexistence des libertés extérieures sous le principe de la liberté universelle : soutenir qu'un créancier possède un droit à exiger du débiteur le paiement de sa dette, cela signifie que la contrainte qui force chacun à agir de cette manière peut coexister avec la liberté de chacun, selon une loi extérieure universelle. Cette loi juridique qui règle la coexistence des libertés au point de vue de la conformité des actions au droit, garantissant à chacun par son pouvoir coercitif une égalité de droits, permet donc d'établir une contrainte réciproque, car ce qui est juridiquement autorisé est par définition ce qui peut instituer une coexistence des libertés. Pour la même raison, la contrainte que l'on oppose à un exercice de la liberté juridiquement interdit, est lui-même légitime, car quiconque empêche à un individu d'accomplir des actes juridiquement autorisés commet envers lui une injustice. Toute cette argumentation repose la proposition selon laquelle le droitet la faculté de contraindre signifient donc une seule et même chose et c'est pourquoi la loi juridique d'une contrainte entretient un rapport analogique « avec la possibilité de libres mouvements des corps sous la loi de l'égalité del'action et de la réaction ${ }^{24}$. Or, il estnécessaire de remarquer que dans cette contrainte réciproque, personne ne se soumet à autrui, mais chacun en se soumettant au tout, en tant qu'il coappartient à l'unité du tout, est en droit d'exiger de la part de l'autre la même soumission à l'égard de la loi juridique, et, par là, il établit une égalitée ${ }^{25}$. Ainsi, la loi qui interdit même de toucher ce qu'autrui possède sous une forme et selon une qualité déterminées, doit accorder à chaque individu la garantie qu'il sera protégé de son côté relativement à ce qui lui appartient : «Je ne puis être lié par la contrainte, insiste Kant, que dans la mesure où je puis tout aussi bien contraindre les autres » sous l'obligation qui me lie à la loi juridique ${ }^{26}$. Parconséquent, l'analogie avecl'influx physiqueest au fondement même dela sociétécivile car celle-ci repose sur le principe de communauté. En effet, au sein d'une société, toutes les parties d'un tout sont coordonnées les unes aux autres au moyen de l'action réciproque (Wechselwirkung) ${ }^{27}$. Pour qu'il y ait union des volontés (unio voluntationis), chaque volonté doit être une partie de la volonté commune et n'être gouvernée que par la volonté de la totalité, car chacune est liée réciproquement à celle des autres. C'est parce que les individus sont liés par le principe de communauté qu'il devient possible

\footnotetext{
23 Prolégomènes à toute métaphysique future qui pourra se présenter comme science, Ak. IV 357, p. 142, note, (vol. II).

${ }_{25}^{24}$ Métaphysique des moeurs, Ak. VI 232, p.481.

${ }_{26}^{25}$ Réflexion n. 7537, Ak. XIX 449, Théorie et pratique, trad. F. Proust, éd. Flammarion, p. 129.

${ }^{26}$ Réflexion n. 7665, cité par L.Guillermit dans sa traduction de Théorie et pratique, note 37, éd. Vrin, 1990, p. 81.

27 Réflexion n. 7548, Ak. XIX 452, F. Proust, Théorie et pratique, p. 128.
} 
d'exercer un droit à l'égard de l'autre, dont les constitutions garantissent les moyens de le faire valoir s'il est compatible avec la coexistence des libertés. Il n'y a donc de corps commun que là où chaque jugement doit avoir la même force de droit que n'importe quel autre, et où tous les membres doivent disposer d'un pouvoir égal de se contraindre les uns les autres ${ }^{28}$.

La portée analogique du principe de communauté s'étend également au domaine de l'éthique qui fournit des maximes pour les actions. Pour Kant, l'individu, qui œuvre à la réalisation de la fin de sa volonté qui réside dans la moralité, doit s'inscrire dans une relation réciproque avec un autre être existant ${ }^{29}$, selon une liaison d'attraction et de répulsion. En vertu de l'amour réciproque, les hommes sont incités à se rapprocher les uns des autres et cela de façon continue $\mathrm{e}^{30}$, avec cette précision toutefois que l'amour ne doit pas être entendu comme sentiment, plaisir ressenti devant la perfection d'autres hommes, ni comme amour de complaisance, car nulle obligation d'avoir des sentiments ne peut nous être imposée par d'autres $^{31}$. Au contraire, il faut le concevoir comme une bienveillance active qui concerne la maxime des actions, consistant à prendre pour fin le bien-être et le salut d'autrui. A ce titre, le devoir qui s'y rattache est la contrainte que la raison impose au sujet de reconnaître cette maxime comme ayant une valeur universelle ${ }^{32}$. Si l'attraction prend la forme de l'amour, la répulsion se fonde sur le principe de respect, par lequel les hommes se trouvent conduits à se maintenir réciproquement à distance. Le respect n'est pas l'expression d'un sentiment issu de la comparaison de notre propre valeur avec celle de l'autre, mais uniquement une maxime qui impose une limitation à l'estime de soi, par la dignité de l'humanité en une autre personne. Or, cette égalité entre l'attraction et la répulsion trouve sa pleine mesure dans l'amitié, considérée dans sa perfection, comme l'union intime de l'amour et du respect puisqu'elle se définit comme «l'union de deux personnes par un amour et un respectégaux et réciproques ॥ $^{33}$. Ce qui est requis pour l'amitié, selon Kant, c'est la juste mesure del'équilibre entre un principe qui commande le rapprochement et celui qui ordonne de se tenir à une distance convenable l'un de l'autre, en soumettant l'abandon réciproque à des principes et des règles qui viennent limiter l'amour réciproque, par des exigences de respect : faute de quoi, cette amitié est menacée par des interruptions, seuls moyens de goûter à la concorde pour ceux qui font de l'amour un affect ${ }^{34}$. Il est donc aisé de voir que l'amitié est un Idéal de sympathie et de communication. En effet, elle n'est pas le résultat d'une union conçue en vue d'obtenir des avantages réciproques, mais elle se révèle comme un devoir d'humanité capable de constituer une communauté de sentiments, à partir de la faculté et la volonté de communiquer réciproquement à propos de ses sentiments, dépassant ainsi la simple réceptivitéà l'égard du sentiment commun de contentement et de souffrance ${ }^{35}$. La première est une capacité libre de participer et en ce sens, elle donne lieu à une communauté de

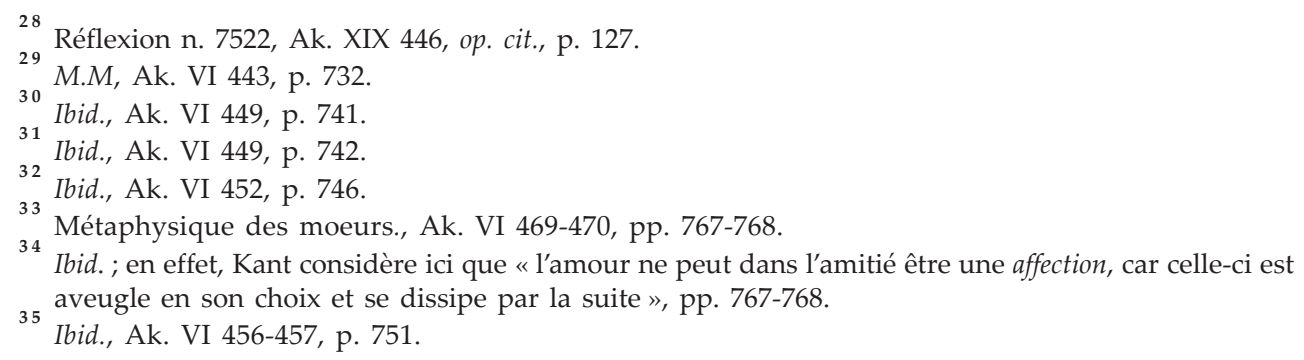


sentiments libérale (communio sentiendi liberalis), alors que la seconde est une capacité communicative non-libre car elle se répand de manière naturelle parmi les hommes qui coexistent ensemble, ce qui établit une communauté de sentiments servile (communio sentiendi servilis). Or, « il n'y a d'obligation qu'en ce qui touche la première » ${ }^{36}$ conclut Kant, car ce n'est jamais une communauté donnée, sinon une tâche à accomplir. Cela explique que l'amitié morale n'est qu'un idéal, un étalon indispensable de la raison normative qui, en effet, a besoin de ce maximum de perfection, pour se donner un horizon et pour estimer le degré et le défaut de ce qui se présente comme incomplet, puisqu'il n'est pas possible, dans la relation de deux individus, de s'assurer dans le sentiment du juste équilibre entre les éléments requis.

Ainsi, force est de reconnaître que la législation de la raison, contrairement à toute interprétation qui la réduirait à un monologisme, s'inscrit dans une réciprocité entre personnes. Sur le plan juridique ou sur le plan éthique, il s'agit bien d'une pensée qui se donne pour exigence de constituer une coexistence des libertés, extérieure d'une part et intérieure d'autre part. Du coup, le formalisme kantien prend tout son sens : certes, la loi morale exclut toute condition empirique mais cela n'exclut pas pour autant tout contenu déterminé. La valeur inconditionnelle livrel'universalité d'une forme qui pousse la personne à agir, dont le projet comporte toujours un contenu, qui est celui d'exercer sa liberté au sein d'une communauté. C'est à partir de l'analogie avec l'égalité d'action et de réaction que ce contenu s'explicite, ceci afin de rendre possible une communauté juridico-civile et une communauté éthico-civile. Toutefois, cette analogie trouve une limite qui est la suivante : à la différence du modèle mécanique, les personnes ne sont coordonnées que dans la mesure où elles obéissent à une autorité supérieure, le souverain dans le domaine juridique et l'être intelligible dans le domaine éthique. Ce qui signifie que la subordination est la condition de possibilité de la coordination, cela parce que toute relation entre personnes exige d'accomplir le passage entre une liberté donnée et une liberté ordonnée : l'interpersonnalité est donc l'enjeu d'un progrès de la raison.

\section{Bibliographie}

Kant, Emmanuel. Oeuvres philosophiques d'Emmanuel Kant, sous la direction de Ferdinand Alquié, Bibliothèque de la Pléiade, Gallimard, Volumes I, II, IIII.

Kant et la philosophie grecque et moderne, en : Actes du Illème congrès de la société internationale d'études kantiennes de langue française, éd. par M. Moutsopoulos, Athènes, 1997.

Revue internationale de philosophie, mars 1988

Recibido: Mayo 31 de 2009 - Aprobado: Julio 19 de 2009

${ }^{36}$ Ibid., p. 751. 